\title{
Währungsverhältnisse in und um Wurzen im Mittelalter und im 20. Jahrhundert
}

\author{
Ewald Hausmann
}

Im 12. und 13. Jahrhundert, in der Periode der regionalen Pfennigwährung, waren Münzen in Mitteldeutschland einseitig geprägte Pfennige, in der Numismatik Brakteaten genannt. ${ }^{1}$ Das Recht, Münzen zu prägen und in Umlauf zu bringen, hatte im Prinzip nur der König. Besaß ein Lehnsmann eigene Silberbergwerke, konnte der König ihm das Münzregal und damit eine Einkommensquelle übertragen. Die Entdeckung des Silbervorkommens zu Beginn des 13. Jahrhunderts im Gebiet des Bistums an der Elbe bei Scharfenberg war deshalb dem Bischof hochwillkommen und dem Markgrafen Anlass, eigene Ansprüche geltend zu machen. Bischof Heinrich konnte jedoch mit Hilfe des Kaisers seine Rechte als Territorialherr am Ertrag der Gruben sichern.

Der direkteste Weg, das Silber zu verwerten, war die Prägung und Ausgabe von Münzen. Der Bergmann erhielt für sein Silber einen festen
Preis, der so weit unter dem Nennwert der geprägten Münzen lag, dass die Prägekosten

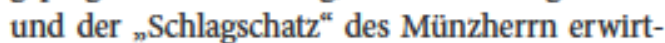
schaftet werden konnten. Der Schlagschatz, der gewöhnlich um ein Prozent betrug, konnte gesteigert werden, wenn man die Münzen mit geringerer als der vorgeschriebenen Masse ausbrachte. Diese Praxis ist auch durch die bischöflichen Pfennige belegt.

Wo das Geld des Bischofs geprägt wurde, ist nicht überliefert. Sein Amtssitz auf der Meißner Burg bot sich schon wegen des gespannten Verhältnisses zum Markgrafen kaum als Münzstätte an. An die Örtlichkeit wurden keine hohen Anforderungen gestellt. Sicherheit für Material und Endprodukt einerseits und möglichst kurze und sichere Wege des Silbers zur Prägestätte andererseits wurden angestrebt. Die seit 995 verfügbare Burg in Wurzen wie das 1219 erworbene

\section{Überlieferte Pfennige von aus der Zeit von 1228 bis 1312}

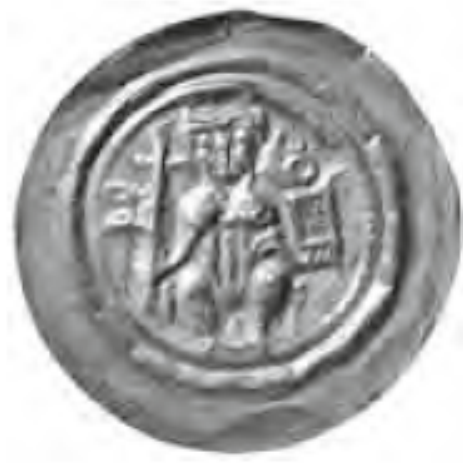

Heinrich I. (1228-1240)

Der Bischof mit Mitra sitzt auf einer Bank, hālt in der Rechten einen Kreuzstab, daneben die Lehensfahne, in der Linken ein Evangelienbuch, darüber zwei Ringel. Neben den geistlichen Attributen war dem Bischof die Fahne als Symbol der Landesherrschaft wichtig.

$[0,84 \mathrm{~g}$, Thieme 1303]

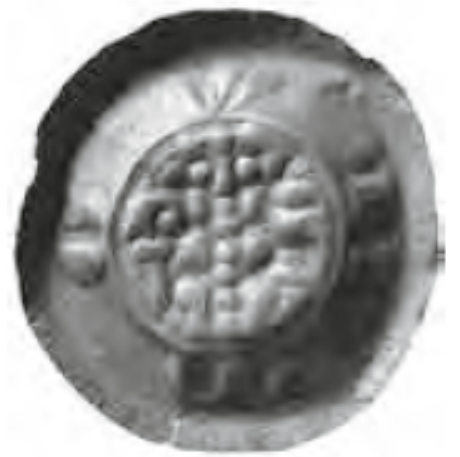

Witigo II. (1266-1293)

Barhäuptiger Bischof sitzt frontal, in der Rechten Krummstab, in der Linken Kelch, am Rand die Buchstaben W N M O. Ein Deutung der Randschrift wäre spekulativ. Die verringerte Masse und das primitivere Bild machen den abnehmenden Wert des Pfennigs deutlich. $[0,74 \mathrm{~g}$, Thieme 1327]

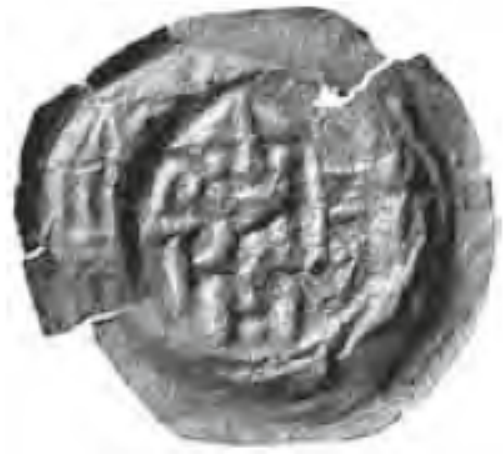

Bernhard II. von Kamenz (1293-1296) oder Albrecht III. von Leisnig (1297-1312) Bischof mit Mitra sitzt auf einer Bank, in der Rechten Krummstab, in der Linken Schlüssel, am Rand beiderseits ein Turm. Masse und Bildqualitāt haben weiter abgenommen. Geblieben ist der Feinheitsgrad des Silbers. $[0,46 \mathrm{~g}$, Thieme 1340] 
feste Haus in Stolpen boten sichere Unterkunft und kämen in Frage.

Da das Erz bereits am Bergwerk verhüttet wurde, waren nur geringe Metallmengen $\mathrm{zu}$ transportieren, deren Sicherung gewährleistet werden konnte. ${ }^{2}$ Die weitere Bearbeitung erforderte keine aufwändigen technischen Einrichtungen. Lediglich die Herstellung der Prägestempel setzte handwerkliche und künstlerische Fähigkeiten voraus. Diese fanden sich unter anderem in "Dienstleistungsfirmen", die verschiedene Münzstätten belieferten. Die Beispiele zeigen, dass mit den Jahren auf künstlerische Gestaltung immer weniger Wert gelegt wurde.

Auf Märkten wie in Wurzen, Mügeln und Bischofswerda galten nur die aktuellen Ausgaben des jeweiligen Territorialherren, hier also die Pfennige des Bischofs. Fremde Ausgaben waren gegen die ortsüblichen einzuwechseln - gegen Gebühr, versteht sich. Außerhalb der Märkte wurden sie nach ihrem Silbergehalt, das heißt nach Gewicht taxiert.

Im ersten Drittel des 14. Jahrhunderts löste der Groschen den Pfennig als Leitwährung ab. Die Prägung der Groschen erforderte größeren technischen Aufwand, den die kleinen Pfennigprägestätten nicht leisten konnten. Die Scharfenberger Gruben lieferten zu dieser Zeit bereits kein Material mehr, da die oberflächennahen Erzgänge erschöpft waren. Beide Gründe beendeten die Ausgabe eigenen bischöflich meißnischen Geldes.

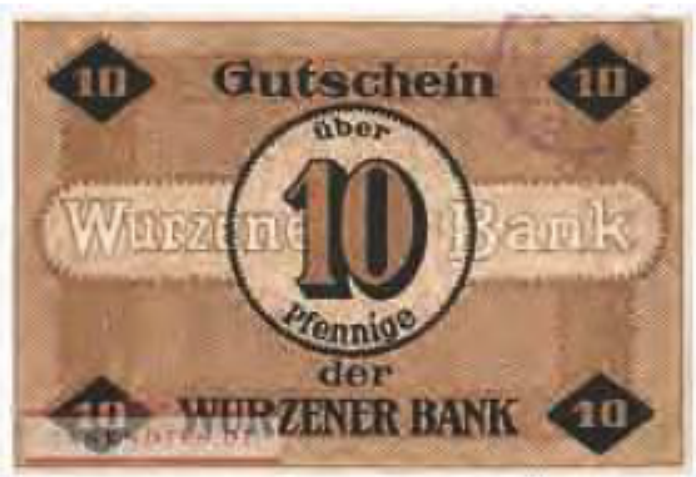

Gutscheine der Wurzener Bank,

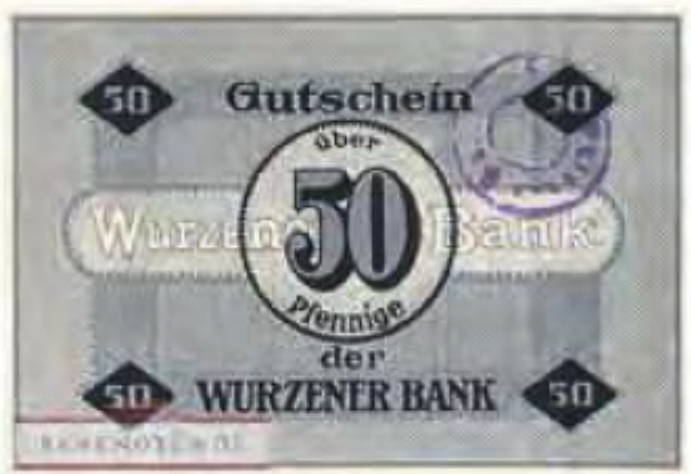

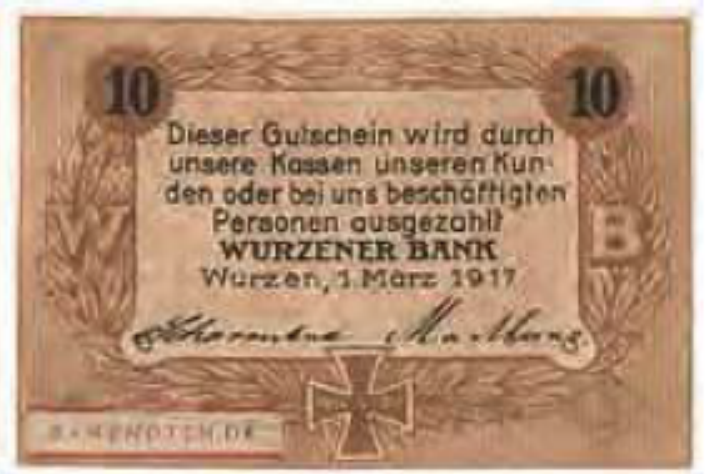

\section{Wurzener Notgeld aus dem}

\section{Jahrhundert}

Nach jahrhundertelanger Pause kam in Wurzen wieder eigenes Geld in Umlauf. Während des Ersten Weltkriegs wurde des Münzgeld knapp. Gold- und Silbermünzen benötigte der Staat zur Bezahlung von Importen. Privatleute reagierten mit Hortung des Edelmetalls. Nickel und Kupfer brauchte die Rüstungsindustrie, deshalb mussten im Verlauf des Krieges Kleinmünzen aus Zink, Eisen und Aluminium hergestellt werden. Die Reichsbank ersetzte die Edelmetallmünzen zu 1, 2, 5, 10 und 20 Mark durch Darlehenskassenscheine, mit gleichen Nominalen, die aber auf Dauer den (unter anderem durch Preiserhöhungen) steigenden Bedarf nicht decken konnten.

Ersatz für die silbernen Fünfziger und die Kleinmünzen aus Nickelbronze bzw. Kupfer mussten die Städte und Gemeinden selbst schaffen, bis die Prägung in Ersatzmetallen in Gang kam. Man achtete aber streng darauf, dass das Geldmonopol der Reichsbank gewahrt blieb. Das heißt, die Scheine waren kein Geld, sondern Gutscheine oder andere Zahlungsversprechen, für die der Herausgeber zu bürgen hatte.

Seit 1919 nahm die Reichsregierung die Hyperinflation in Kauf, um die Kriegsschulden abzubauen. Der Wert der Mark verfiel bald so rasch, dass Reichsbank und Länderbanken nicht mehr in der Lage waren, rechtzeitig genügend Zahlungsmittel zur Verfügung zu stellen. Wieder

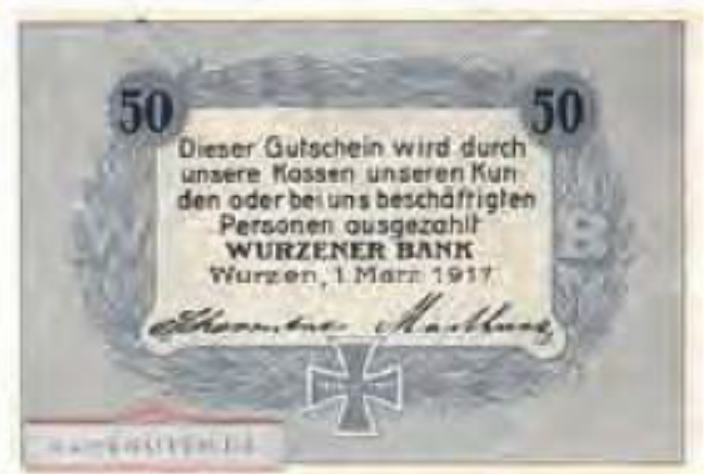


mussten die Kommunen mit eigenen Ausgaben aushelfen. Am Ende gaben Banken und Firmen neue Nominale so schnell aus, dass sie wenigstens einmal zum Bezahlen dienen konnten, bevor sie wertlos wurden.

Der Betrag von 2 Millionen (Papier-)Mark hatte am 17. August den Kurswert von 0,72 Dollar. Am 1. September betrug der Kurswert noch 0,19 Dollar, um in der Folgezeit noch schneller abzunehmen: auf 0,5 Millionstel Dollar am 29. November, dem Höhe- und Endpunkt der Inflation.

Die Braunkohlengrube "Herrmannsglïck ${ }^{*}$ in Fremdiswalde, jetzt ein Ortsteil von Grimma, verwendete Schecks, deren Formulare mit jeweils aktuellen Daten versehen werden konnten. Sie wurden praktischerweise bei der Wurzener Bankfiliale, der am schnellsten erreichbaren, eingelöst.

Wertpapiere wie Aktien als eine Art Sachwert überstanden die Inflation wenigstens $\mathrm{zu}$ einem großen Teil. Die Aktie der Sächsischen Broncewarenfabrik AG Wurzen ist ein eindrucksvoller Beleg dafür. Am Ausgabetag 1. Juni 1922 stand der Kurs der Goldmark bei 65 Papiermark. Der Wert der Aktie betrug demnach am Ausgabetag 1.200 Goldmark bzw. 78.000 Papiermark. Ein am 1. Juni 1922 errichtetes Bankkonto von 78.000 Papiermark war am Ende der Inflation praktisch Null, die Aktie aber 200 Goldmark wert, wie der rechteckige rote Stempel angibt. Der kleine runde Stempel besagt, dass die Dividende für 1922 ausgezahlt wurde. Der Betrag ist leider nicht zu ermitteln.

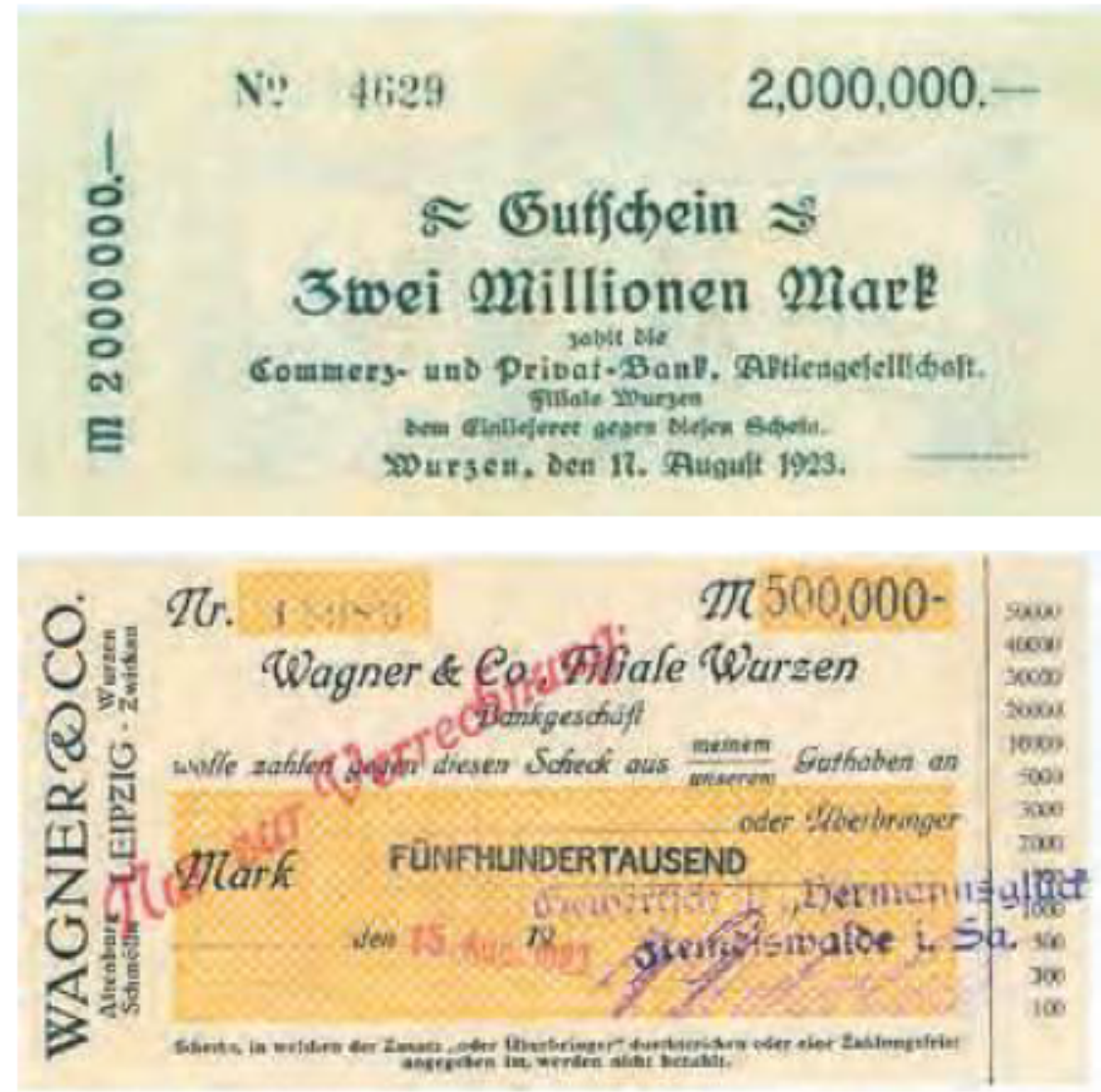

Die 1862 gegründete Firma produzierte 1945 bis 1972 als Bronzewarenfabrik Neidhard \& Zimmermann und 1972 bis 1990 als VEB Spezialleuchtenbau, danach als SBF Spezialleuchten Wurzen. Seit 2012 ist die Produktion nach Leipzig verlagert.
Gutscheine der Commerz- und Privatbank AG, Niederlassung Wurzen, 1923

Scheck der Gewerkschaft "Herrmannsglück" über 500.000 Mark, 1923

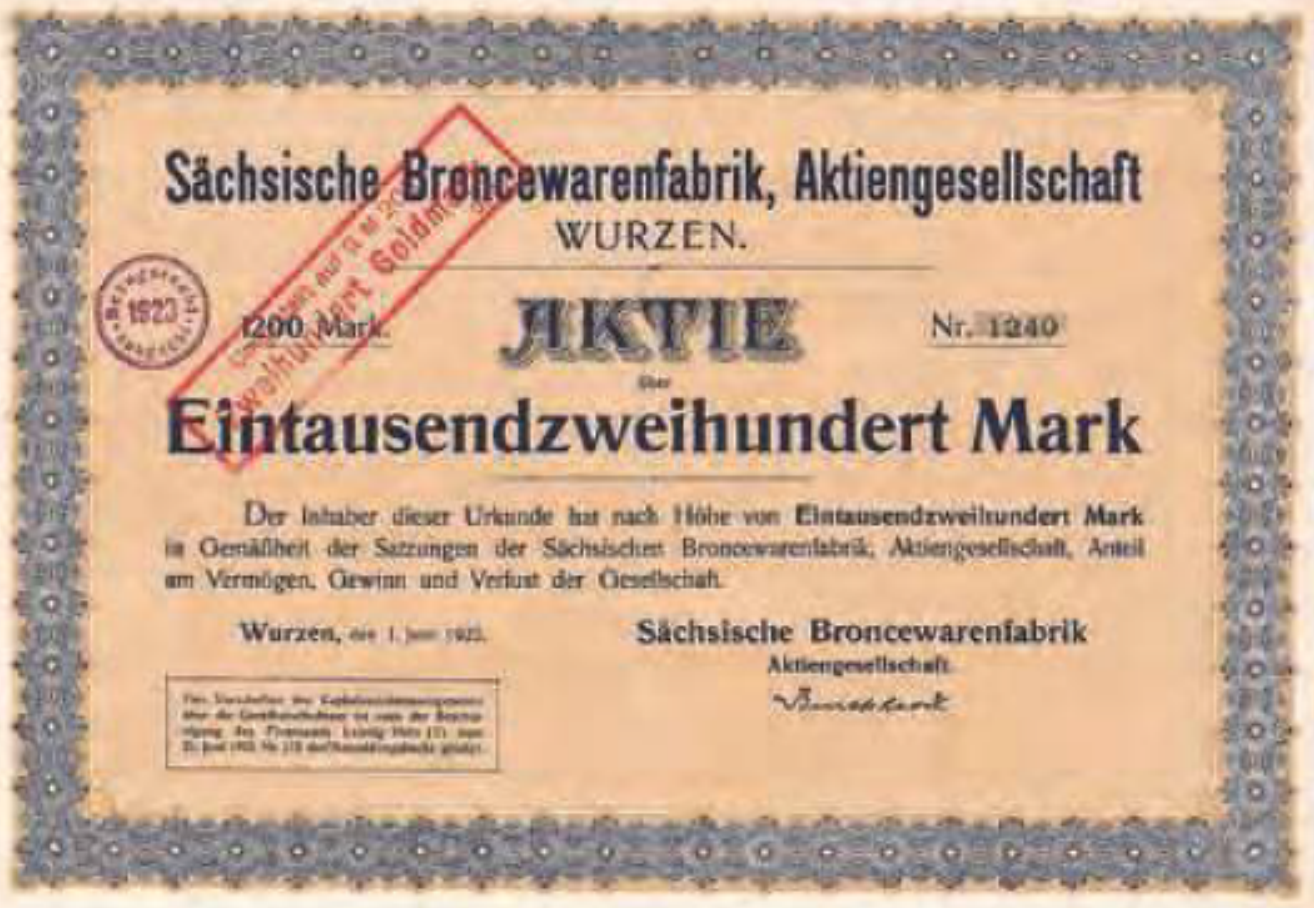




\section{Medaillen bewahren die Erinnerung an Personen und Ereignisse in Wurzen ${ }^{3}$}
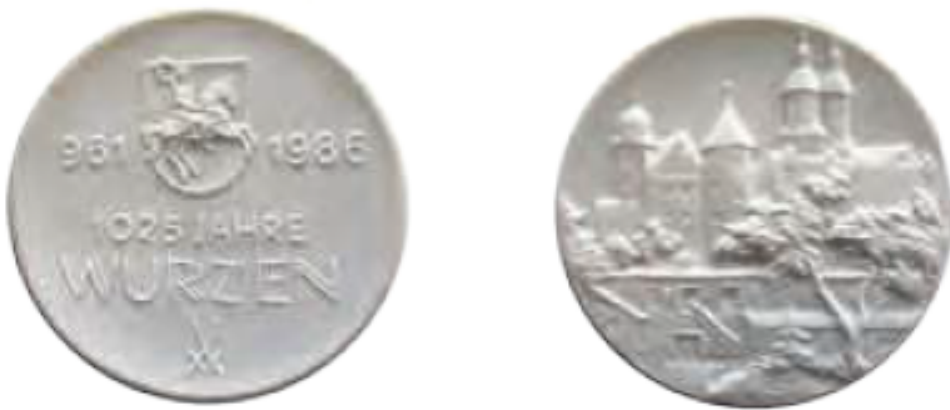

Porzellanmedaille auf die 1025-Jahr-Feier Wurzens von 1986. ${ }^{4}$ Das Stadtwappen steht auf der Vorderseite zwischen den Jahreszahlen 961 und 1986, darunter der Anlass der Ausgabe über den MeiBner Schwertern. Auf der Rückseite findet sich der Gebäudekomplex aus Schloss, Dom und ehemaligem Kornhaus. Es existiert eine zweite Medaille auf den gleichen Anlass mit mehrzeiligem Text auf der Rückseite.
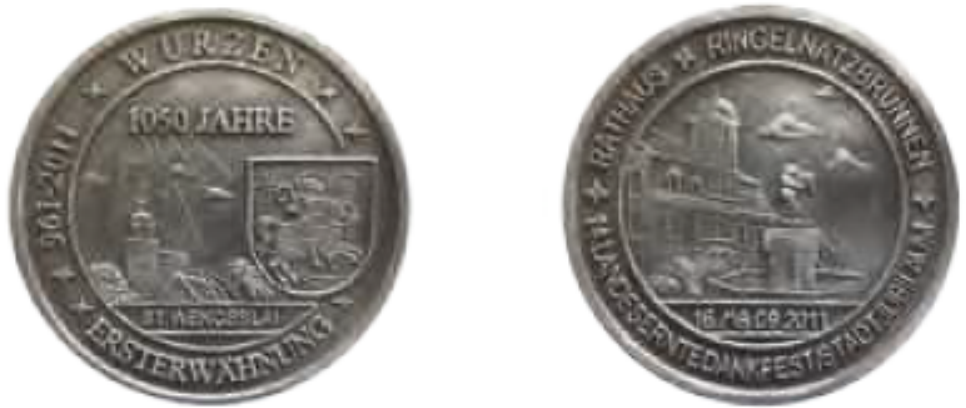

Zinnmedaille auf die Feier des 1050-jährigen Bestehens Wurzens von 2011. Die Vorderseite teilt sich die Wenceslai-Kirche mit dem Wappen in einer älteren Form. Die Umschrift nennt den Ausgabeanlass. Auf der Rückseite steht der Ringelnatzbrunnen vor dem Rathaus. Die untere Umschrift besagt, dass das Stadtijubiläum gemeinsam mit dem Landeserntedankfest gefeiert wurde. Es existiert eine zweite Zinnmedaille von Bartel zum gleichen Anlass mit verändertem Motiv.
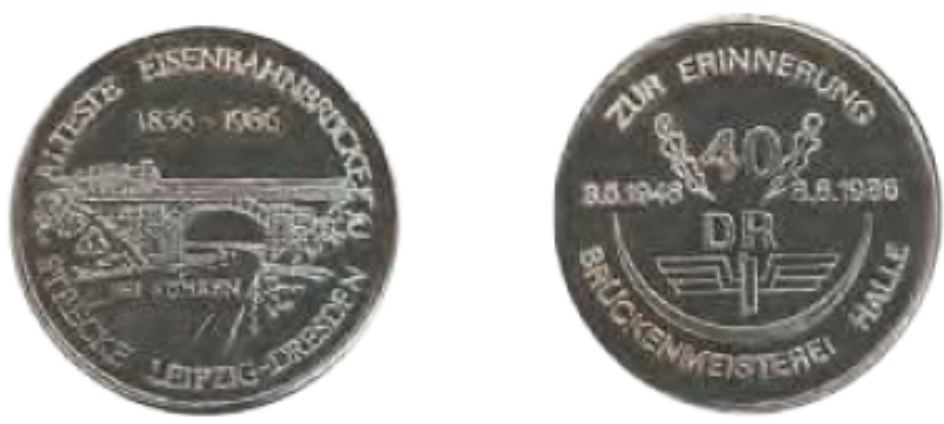

Neusilbermedaille auf die Restaurierung der ältesten Eisenbahnbrücke Deutschlands von $1986 .{ }^{5}$ Zum 150 -jährigen Bestehen der ersten Eisenbahnbrücke Deutschlands wird sie von einer Lok befahren vorgestellt. Der Ort Kühren, bei dem sie die B 6 überquert, gehört jetzt zu Wurzen. Auf der Rūckseite feiert die Brūckenmeisterei Halle, die die Brücke so gründlich sanierte, dass noch immer Züge darüber rollen kōnnen, ihr 40 . Jubiläum.

3 Die Medaillen (außer Nr. 6) stellte A. Brade, Wurzen, bereit.

4 Vgl. Karl-Heinz Weigelt: Medaillen aus Meißner Porzellan. Bd. 6 (1984-1987). Berlin 1988, Nr. 8.443.

5 Vgl. H. Huschka: Medaillen aus der Konsumgūterproduktion VEB Walzwerk Hettstedt. St. Valentin 2014, S. 325, Nr. 280 .
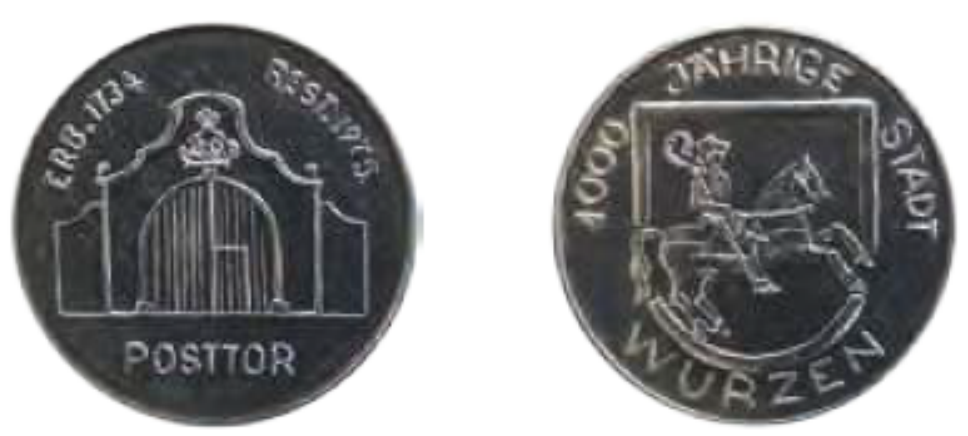

Neusilbermedaille auf die Bewahrung und Restaurierung des barocken Tores der alten Posthalterei durch die Initiative von Herm Artur Ziegert von 1975. Die Vorderseite stellt die Umrisse des Tores dar und nennt die Daten des Baus und der Restaurierung. Die Rückseite wird vom Stadtwappen ausgefült, dessen Rahmen der Reiter sprengt. 

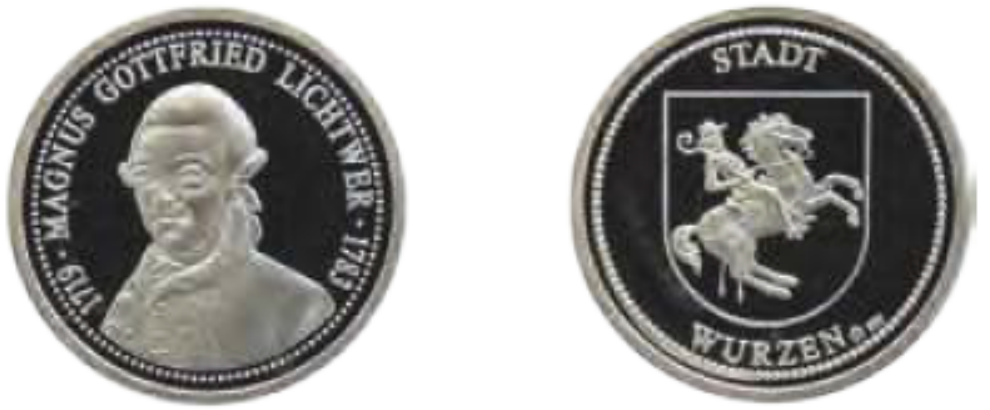

Der Fabeldichter, Hochschullehrer, Richter und Kommunalpolitiker Magnus Gottfried Lichtwer, geboren 1719 in Wurzen, gestorben 1783 in Halberstadt, gilt als eine der bedeutendsten Wurzener Persönlichkeiten. Er wurde in jüngster Zeit als Namenspate eines Gymnasiums und mit einer Siber-Medaille geehrt.

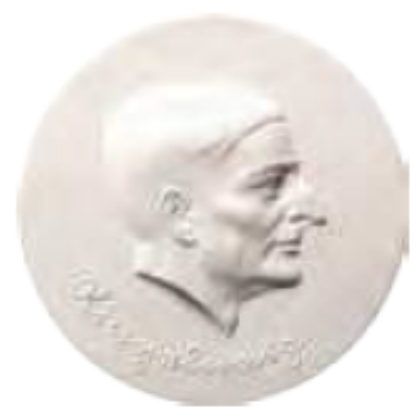

Dem Bildhauer Johannes Henke verdanken wir die Porzellanmedaille zur Ehrung des bekanntesten Sohnes der Stadt Hans Bötticher, der sich Joachim Ringelnatz nannte. ${ }^{6}$ Geboren 1883 in Wurzen, gestorben 1934 in Berlin. Dichter, Humorist, Kabarettist, Maler, Matrose, Soldat, Lebenskūnstler. Die Rūckseite trägt das Firmenzeichen der KPM. Henke schuf daneben auch eine Medaille in Bronze. Die Staatliche Porzellanmanufaktur MeiBen brachte 2004 ebenfalls eine Portrātmedaille auf Ringelnatz heraus.
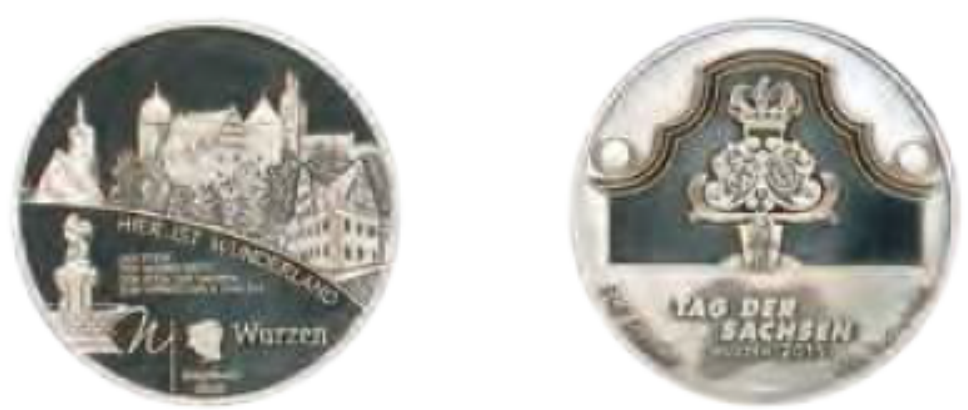

Die Silbermedaille der Sächsischen Numismatischen Gesellschaft auf den "Tag der Sachsen"7 stellt auf der Vorderseite das Gebāudeensemble von Schloss und Dom vor, auf der linken Seite ergänzt um die Wenzelaikirche. Im Abschnitt der Ringelnatzbrunnen und der Kopf von Joachim Ringelnatz als Silhouette. Dazu der Spruch des Dichters: „Der Stein der Weisen sieht dem Stein der Narren zum Verzweifeln ähnlich." Auf der Rückseite steht im Feld der Torbogen der alten Posthalterei mit der Kōnigskrone über den Wappen von Polen und Sachsen. Im Abschnitt das Logo des Kuratoriums „Tag der Sachsen" und der Name Herausgebers der Emission: SNG DRESDEN.
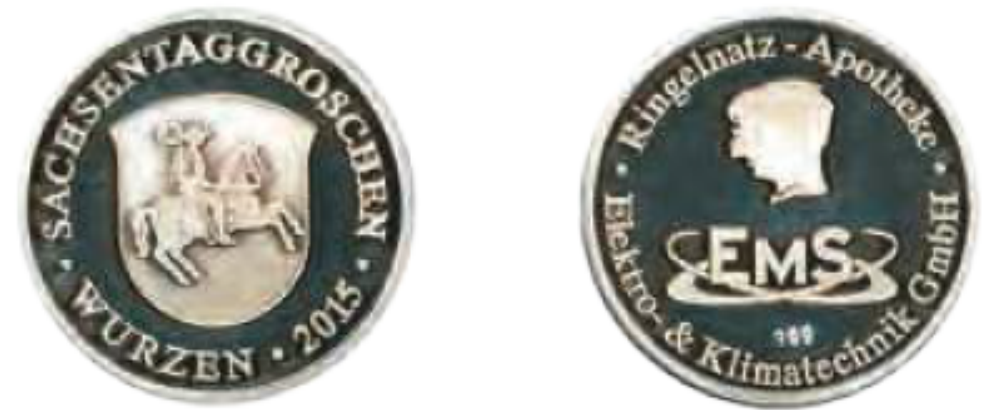

6 Vgl. B. Weisser: Das Medaillenwerk von Johannes Henke, in: G. Dethlefs u.a. (Hrsg.), Westfalia Numismatica 2013. Festschrift zum 100jährigen Bestehen des Vereins der Münzfreunde für Westfalen und Nachbargebiete. 2013, S. 377-378, Nr. 34. Ich danke für die freundliche $\mathrm{Ge}$ nehmigung des Autors.

7 Vgl. R. Reimann, 24. Tag der Sachsen in Wurzen, in: Die Medaillen der Sächsischen $\mathrm{Nu}$ mismatischen Gesellschaft e. V. 1989-2015, S. 144, Nr.6.1.24.1.

8 Vgl. R. Reimann, 24. Tag der Sachsen in Wurzen, in: Die Medaillen der Sächsischen Numismatischen Gesellschaft e. V. 1989-2015, S. 157, Nr. 6.2.17.1.

Autor

Ewald Hausmann

Lichtenau

Die Vorderseite der Auswurfmedaille trāgt das Wappen der Stadt und die Bezeichnung SACHSENTAGGROSCHEN WURZEN 2015. Die Rückseite ist den Namen der Sponsoren der Ausgabe und ihren Logos vorbehalten. 